\title{
Real-Time ECG Monitoring Device With Heart Pulse Sensor
}

\author{
Safiya Khatoon ${ }^{1}$, Syed Sibtain Khalid ${ }^{2}$, Waquas Tariq ${ }^{3}$, Safdar Tanweer ${ }^{4}$, Naseem Rao ${ }^{5}$ \\ \{safiyakhatoon288@gmail.com, s.sibtainkhalid@jamiahamdard.ac.in, safdartanweer@gmail.com, \\ naseemjmi0786@gmail.com\}
}

Department of Computer Science \&Engineering (SEST), Jamia Hamdard, New Delhi-110062

\begin{abstract}
In this paper, We have analysed the real-time acquisition of ECG signals and concatenated with the intelligent devices Arduino for better service for the desired pupils suffering from heart misfunctioning. The ECG signals are processed, stored and transmitted to the target location in real-time. It can be processed offline as well as online. However, the main aim to review the current state of the Monitoring Device where it extensive depth analysis to finding in the area of smart health Monitoring for the ease of patient and the doctors. Moreover, the paper concludes that our health Monitoring Device will be prevalently implemented with home environment monitoring and control systems and the sensor ( AD8232) is usually do for a few minutes and it is a painless or help to diagnosis much common heart problem of all age peoples.
\end{abstract}

Keywords: Electrocardiograph, electrode, sensors, health monitoring devices, Arduino.

\section{Introduction}

In the present time, people suffering from heart diseases are increasing so much. In India, many lives are affected because of a lack of knowledge in diagnostic awareness. The electrocardiogram (ECG) is the most commonly used diagnostic test for measuring the electrical activity of the heartbeat in the medical field. It was discovered by William Eintheon in 1895.It is a diagnostic tool that is used to measure and record the electric activity related to cardiac contraction of the heart in fastidious detail. it is a non-invasive, painless test with quick results. With the help of this information, we can easily get to know about the conditions of the heart's status. These conditions can vary from minor to life-threatening. It is an easy and affordable test that is used generally in the assessment of the patient with pain in the chest with zero risks to the patient. The ECG is the cornerstone for making the decision for eligibility for thrombolytic therapy. An ECG is basically a chronicle of the electrical movement on the body surface produced by the heart muscles. An ECG info is collected by electrodes placed at the selected location on the patient's body then it shows the result In the form of ECG waveform on a monitor window. Most of the ECG devices are very expensive. There are some low coast devices that are also available but they are not much accurate. Most hospitals and diagnostic centres in India use incorporated devices designed to measure the body pressurecirculatory strain and pulse of the patient. Although such devices are valuable, their cost is usually uneconomical. This paper depicts the design of an ECG monitoring system which monitors ECG subject by using Arduino ECG sensor ad823 module and heartbeat pulse sensor we sticking an electrode On the arms and then showing the ECG on the monitor window. 


\section{Literature review}

Ashlesha A. Patil et al [2].implementation of IoT based smart health care system which is used for the purpose of patient health monitoring and chose a cloud server to save data and transmitted to the PC and versatile for family and specialist reference

Matthew D'Souza et al [3].it informs the data accessed and control by a handheld device operated by medical participations it explores and compares the performance of real-time acquisition for patient ECG signal

Daniel Lucani et al [5]. gadget can get, store and transmit ECG sign to PC base stage or exceptionally arrange with web abilities to remote observing stations Mirza Mansoor Baig et al [9].a review of the smart health monitoring system and overview of there design and modelling

\section{Methodology}

\section{Block Diagram Represent of ECG Signal Condition or Filtration}

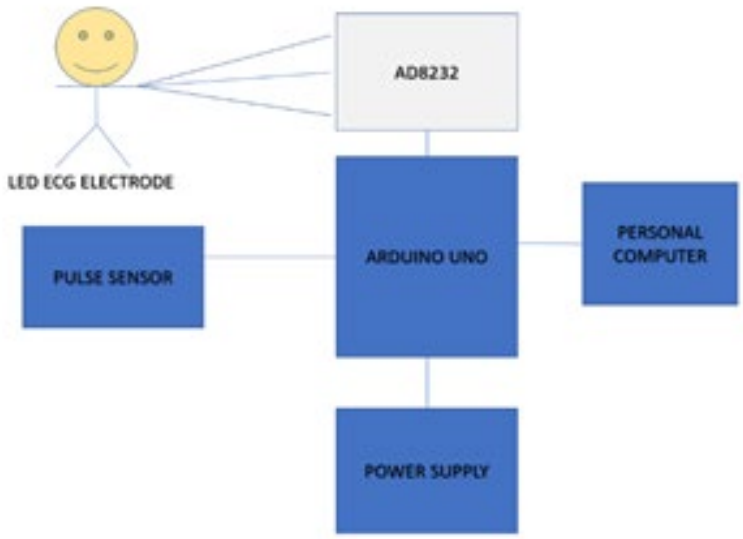

Fig.1 Block diagram

Here the ECG signal which has 3 electrodes connected with the AD8232 ECG module and pulse sensor with Arduino micro-controller to processing the biometric signal from the human body. Arduino is responsible for the operation it will check all the inputs are proper or not then it shows the "Normal" or "Abnormal" activity of the heart.

\section{Signal Acquisition Stage}

The AD8232 module breaks out nine associations, for example, SDN, LO+, LO-, OUTPUT, $3.3 \mathrm{~V}$, GND outfit central pins were work with an Arduino or other improvement board. These sheets are RA (Right Arm), LA (Left Arm), and RL (Right Leg) pins to join and use your own one of a kind custom sensors. In addition, there is a LED marker light that will throb to the rhythm of a heartbeat. The AD8232 is a planned sign moduling deter for ECG and other biopotential estimation applications. It is expected to separate, upgrade, and channel little biopotential signals inside seeing boisterous conditions, for instance, those made by 
development or remote anode course of action. This is a monetarily wise board used to measure the electrical development of the heart. This electrical development can be laid out as an ECG. ECGs can be extremely uproarious, the AD8232 Single Lead Heart Rate Monitor goes about as an activity amp to help get a sensible sign from the PR and QT Intervals successfully.

\section{Application}

AD8232 is an incorporated sign moduling hinder for ECG which configuration to separate intensify, and channel little biopotential

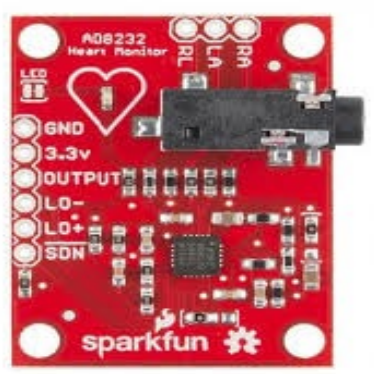

Fig 3. ECG Module (AD8232)

\section{Pulse Sensor}

Heartbeat Sensor is a well-organized fitting and-play beat sensor for Arduino essential thing heartbeat sensor involves a light-transmitting diode and a locator like a light perceiving resistor or a photodiode. The heartbeat thumps cause an assortment in the movement of blood to different zones of the body. The proportion of light held depends upon the blood volume in that tissue It. in light of the of photoplethysmography where heart beat rate is to be checked, the arranging of the thumps is progressively critical.

\section{Application}

- This sensor is used for sleep tracking or we can say that live heart information into the monitoring window

- This sensor is used for anxiety monitoring with having there optical amplifying and noise elimination.

- For health band is used and this sensor work by the fingertip which is connected with the Arduino board.

\section{Signal Conditioning Stage}


Arduino Uno is a microcontroller to install the program.there are many types of Arduino that have their own different software. Here we were using an 8-bit microcontroller board called AT mega 328 which is having 14 digital input/output. The atmega328 contains a 10-piece dynamic theory ADC with testing repeat running from $10 \mathrm{kHz}$ to $200 \mathrm{kHz}$

The force pins are as per the following: Vin - The information voltage to the Arduino and supply voltage can utilize this pin. $5 \mathrm{~V}-$ This pin yield a managed $5 \mathrm{~V}$ from the controller on the board. $3.3 \mathrm{~V}$ - A $3.3 \mathrm{~V}$ stockpile created by the on-board controller. The most extreme current draw is $50 \mathrm{~mA}$. GND - Ground pin

\section{Application}

- This is used for making obstacle avoiding robot

- Automatic opening dustbin using ultrasonic sensor

- For making Drones we use this application

- For home automation or security device we used this application

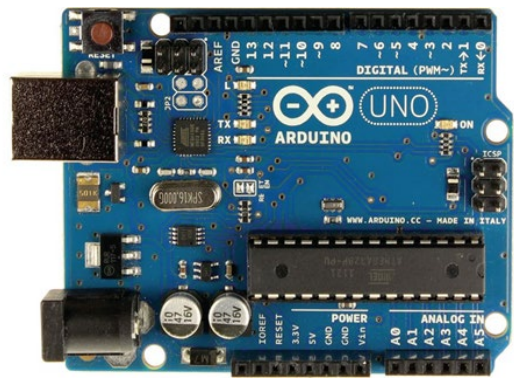

Fig 4 Arduino Uno

\section{Result and Discussion}

We put the electrode on the human body that will sense the normal and abnormal activity which shows by the help of Arduino and sensor on the monitor window in the waveform of the ECG signal.

The output connection is shown in fig 


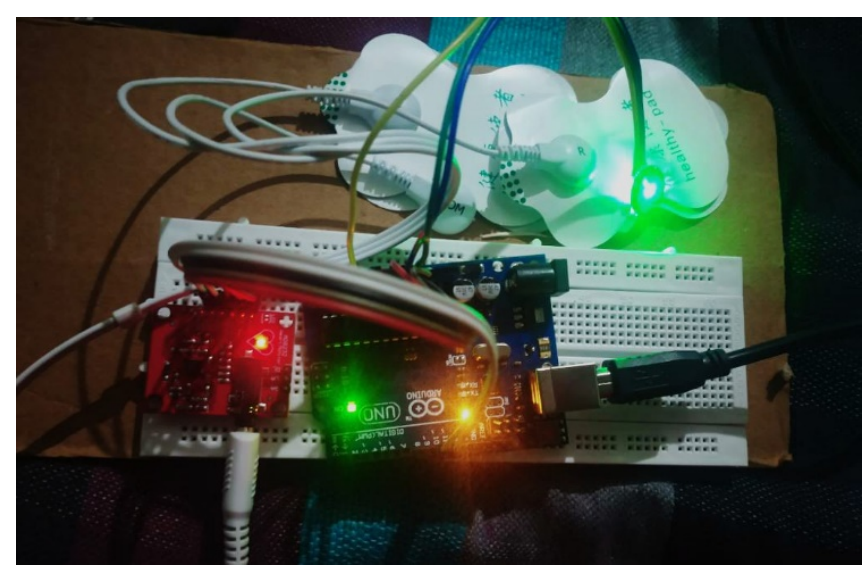

Fig 5. Picture of circuit

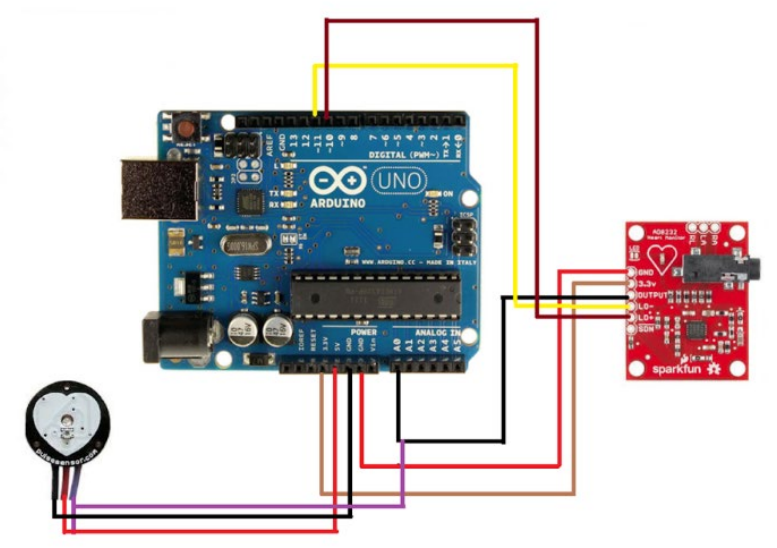

Fig 6. Connection circuit

The typical ECG extend is $120-200 \mathrm{~ms}$. it is up to $120 \mathrm{~ms}$ where 3 little square on ECG paper anyway QT interim which estimated from the main diversion of QRX complex and with T wave. 


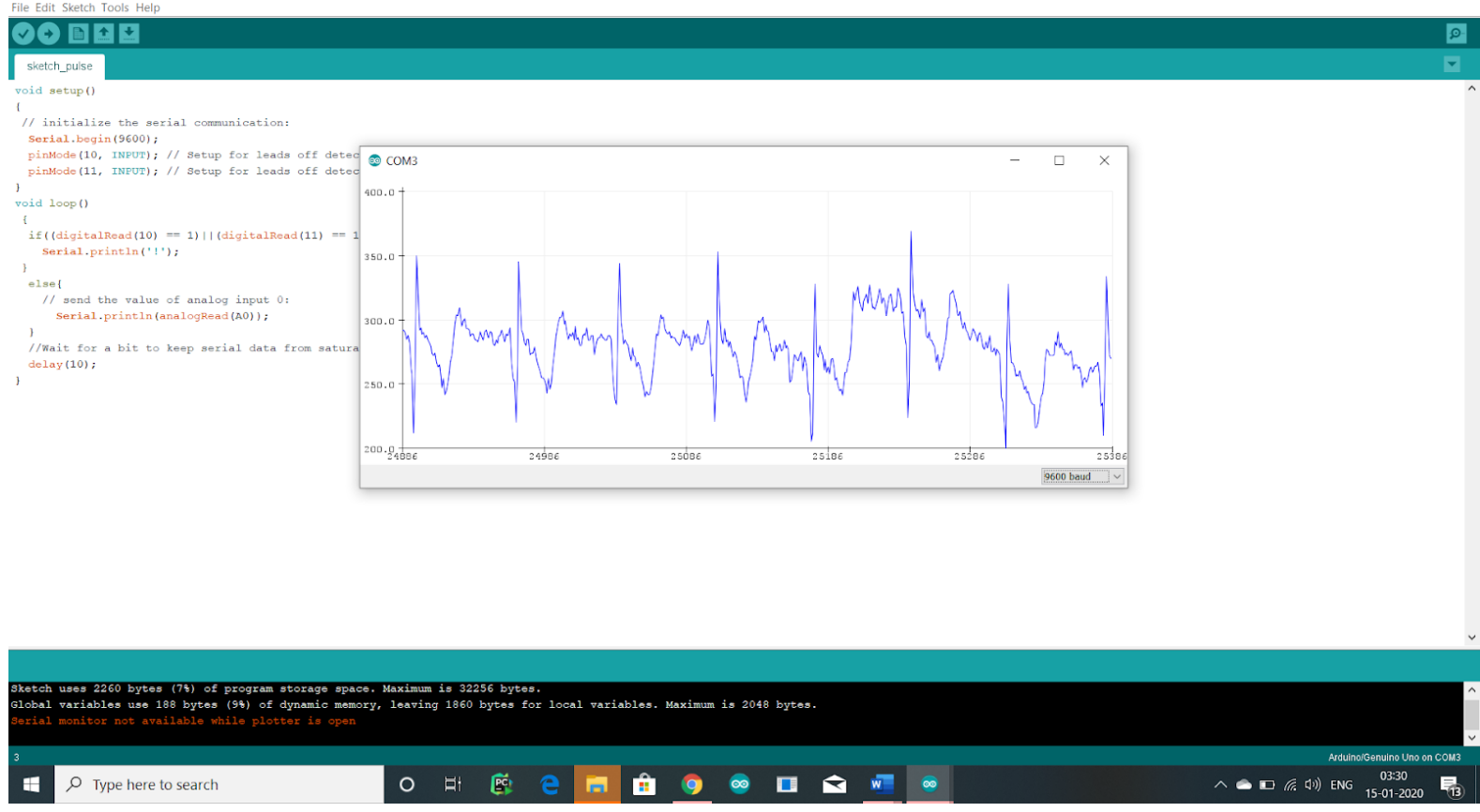

Fig 7.output screen of ECG normal signal

The electrical activities in ECG which show now and then an ECG irregularity is a typical variety of heart mood which doesn't influence your wellbeing. Where it will increase or disturbed the signal get fluctuated which shows some the emergency of heart attack

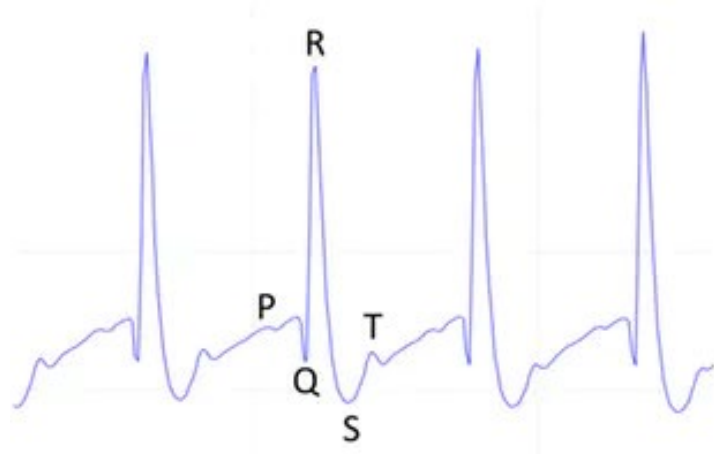

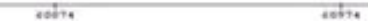
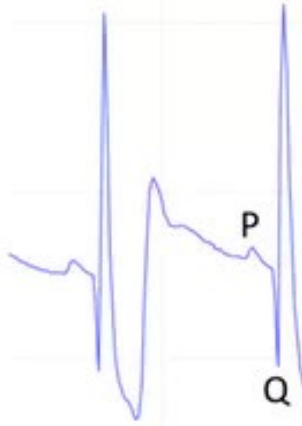

Q

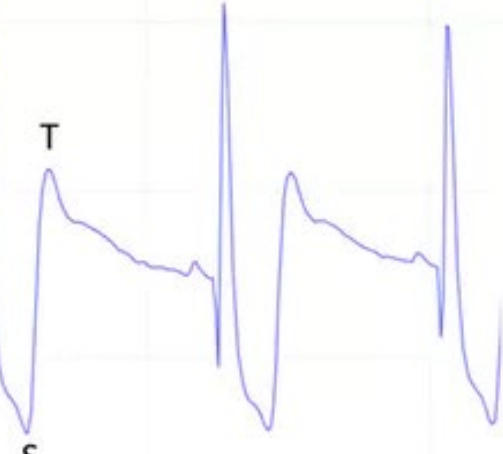

A⿱乛龰⿱中⿰㇀丶㇀ 
Fig 8 . The output of the abnormal waveform

Abnormal pulse rate is diagnosed by ECG readout where some irregular heartbeat rhythm maybe low 40-60 bpm

Due to poor blood circulation in the body which cause the heart rhythm inappropriate or. due to that dizziness, palpitation founding in the chest, etc they may even cause sudden cardiac death

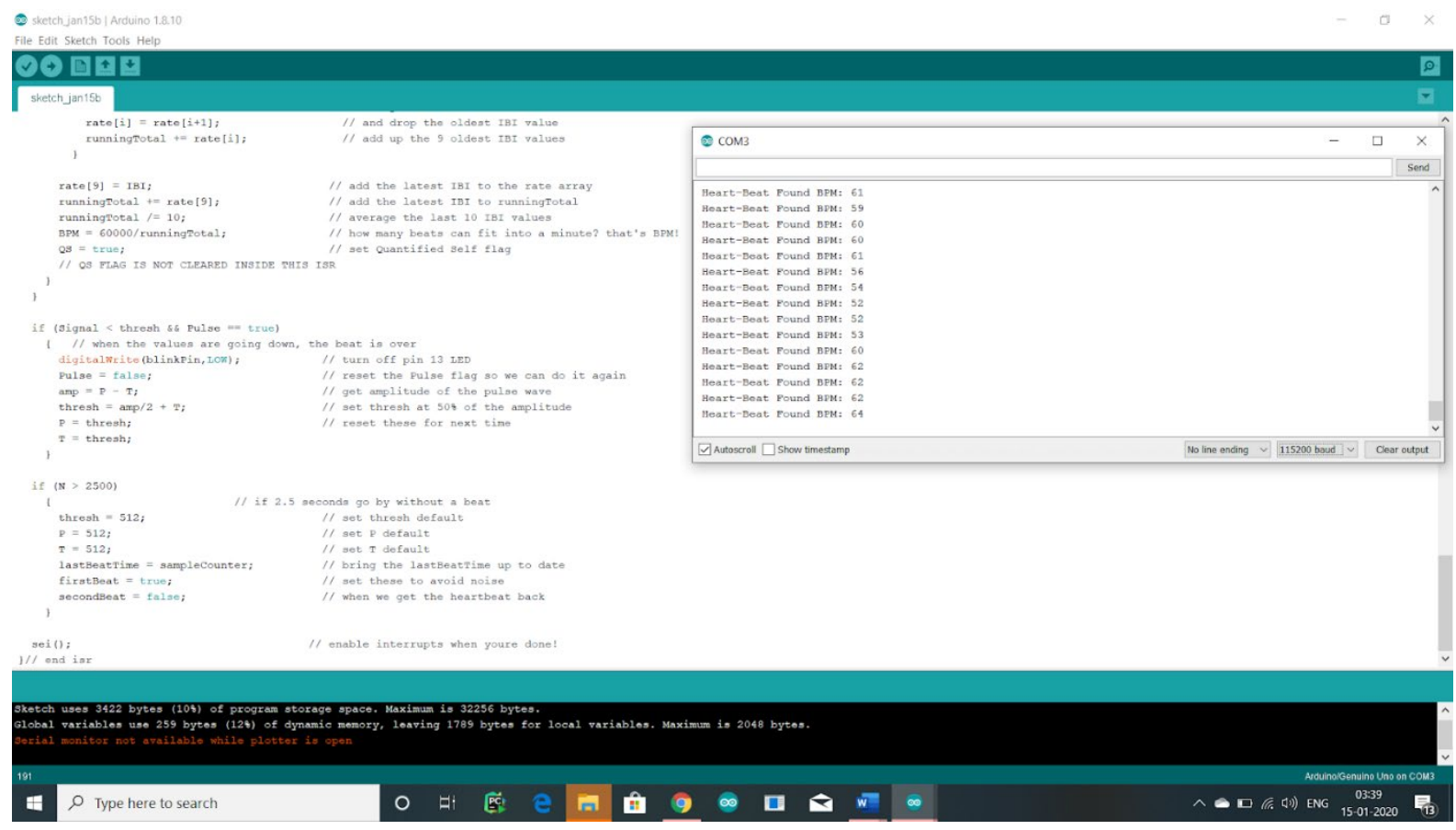

Fig 9. Abnormal pulse rate

The normal pulse rate of the children (age 6 to 15) 70-100bpm

For an adult (age 18 and over) 60-100bpm 


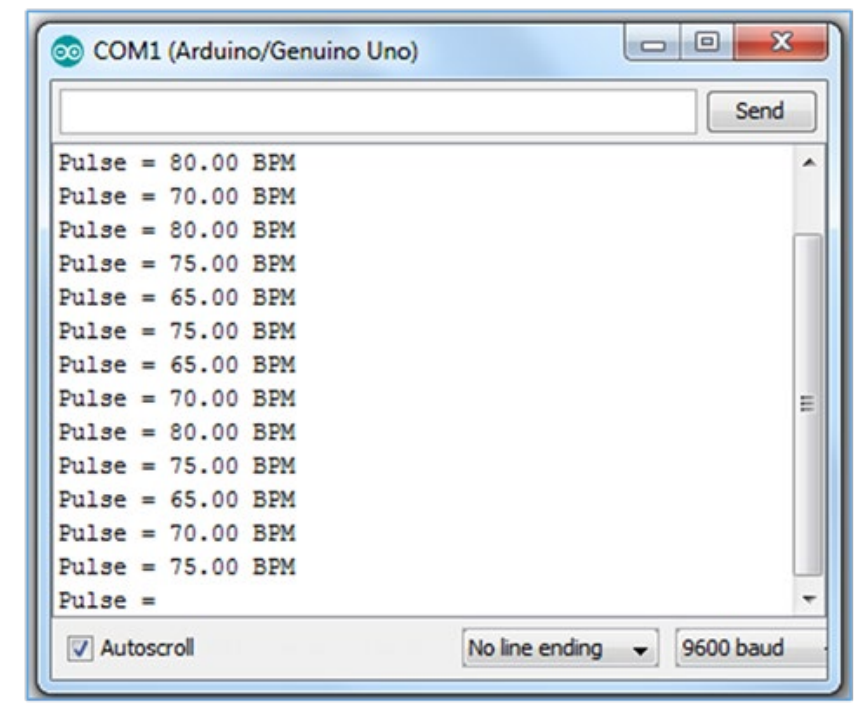

Fig10. Normal pulse rate

\section{Conclusion and Future Prospect}

This experiment result shows on the basis of the signal analysis that the ECG signals can be filtered and will be monitored with cheaper price, much accurate in readings, user-friendly and very easy to use for the common people. We can enhance this system by using IoT base to make it more intelligent we can make an Android app which tells us about the situation of our heart and whenever it is abnormal it alerts the doctor as well as patient.

\section{References}

1. IoT Based Smart Healthcare Monitoring System for Rural/Isolated Areas A.Yogaraj1, M.R Ezilarasan2, Dr. Anuroop.R.V3, C.S.Sivanthiram4, Sunil Kumar Thakur5 1Dept. of ECE, Veltech Dr. RR \& Dr.SR University, Chennai,

2. REVIEW ON-IOT BASED SMART HEALTHCARE SYSTEM Ashlesha A. Patil ME Student, E\&TC Department, SSBT's COET, Bambhori, Jalgaon, India Dr. S. R. Suralkar Professor \& H.O.D. E\&TC Department, SSBT's COET, Bambhori, Jalgaon, India

3. Wireless Medical Information System Network for Patient ECG Monitoring Matthew D'Souza School of Information Technology and Electrical Engineering, The University of Queensland, Brisbane QLD Australia

4. A High-Performance Reconfigurable Hardware Platform for Digital Pulse Processing João M. Cardoso, J. Basílio Simões, Carlos M. B. A. Correia, A. Combo, R. Pereira, J. Sousa, N. Cruz, P. Carvalho, and C. A. F. Varandas 
5. A REVIEW PAPER ON SMART HEALTH CARE SYSTEM USING INTERNET OF THINGS Zankhana Mehul Kalarthi PG Student, Electronics and Communication, GTU, Gujarat, India

6. Real-Time Patient Health Monitoring and Alarming Using Wireless-Sensor-Network Kasim M. Al-Aubidy, Ahmad M. Derbas. \& Abdullah W. Al-Mutairi Intelligent \& Embedded Systems Research Group, Faculty of Engineering, Philadelphia University

7. AFC-ECG: An Adaptive Fuzzy ECG Classifier Wai Kei Lei1, Bing Nan Li1, Ming Chui Dong1,2, and Mang I Vai2 1 Institute of System and Computer Engineering, Taipa 1356, Macau 2 Dept. Electrical \& Electronic Engineering, FST, University of Macau, Taipa, Macau

8. Developing Residential Wireless Sensor Networks for ECG Healthcare Monitoring Nilanjan Dey, Amira S. Ashour, Fuqian Shi, Senior Member, IEEE, Simon James Fong, and R. Simon Sherratt, Fellow, IEEE

9. Smart Health Monitoring Systems: An Overview of Design and Modeling Mirza Mansoor Baig \& Hamid Gholamhosseini

10. Multi-stage Real-Time Health Monitoring via ZigBee in Smart Homes S. Dagtas , ‘ 1 , G. Pekhteryev2, Z. S, ahinoglu `2, 1Department of Information Science, University Arkansas, Little Rock, AR 2Mitsubishi Electric Research Labs, 201 Broadway Ave., Cambridge, MA

11. A portable ECG monitoring device with Bluetooth and Holter capabilities for telemedicine applications Daniel Lucani, Student Member, IEEE, Giancarlo’s Cataldo, Student Member, IEEE, Julio Cruz, Guillermo Villegas, and Sara Wong

12. Issues in Wearable Computing for Medical Monitoring Applications: A Case Study of a Wearable ECG Monitoring Device Thomas Martin, Emil Jovanov, Dejan Raskovic The University of Alabama in Huntsville Department of Electrical and Computer Engineering Huntsville, AL 35899

13. Resource-aware secure ecg healthcare monitoring through body sensor networks honggang wang, university of massachusetts, dartmouth dong ming peng, wei wang, and hamid sharif, university of nebraska-lincoln hsiao-hwa chen, national cheng kung university ali khoynezhad, university of nebraska medical center. 